\title{
Uma Metodologia de Precificação Dinâmica para Produtos e Serviços em Smart Cities
}

\author{
Breno Santana Santos Vinícius Campos Tinoco Ribeiro \\ Ivanovitch Silva
}

\author{
Universidade Federal do Rio Grande do Norte \\ Natal, RN, Brasil, (e-mails: breno_mtb@ufrn.edu.br, \\ vinihcampos@ufrn.edu.br,ivan@imd.ufrn.br).
}

\begin{abstract}
Smart economy is a new economic paradigm for smart cities, allowing integration among all economic sectors to promote greater competitiveness between companies, productivity and cost-benefit for its citizens. Thus, various technological resources have been employed, with emphasis on pricing approaches, which are applied in several contexts (oil, stock exchange, precious metals, among others). However, these mechanisms are little explored in the conjuncture of daily life's products/services, mainly with respect to the use of Electronic Consumer Invoice (NFC-e) as data source. Given this scenario, this paper aims to propose a methodology for dynamic pricing of daily life's products/services. In addition, an initial platform was developed, which was validated by a proof of concept performed in the state of Rio Grande do Norte, evidencing its viability with encouraging results.

Resumo: Smart economy é um novo paradigma econômico para smart cities, permitindo a integração entre todos os setores econômicos a fim de promover maior competitividade entre empresas, produtividade e custo-benefício para seus cidadãos. Para isso, diversos recursos tecnológicos têm sido empregados, com destaque para as abordagens de precificação, as quais são aplicadas em diversos contextos (petróleo, bolsa de valores, metais preciosos, entre outros). Contudo, esses mecanismos são poucos explorados na conjuntura de produtos/serviços do dia-adia, principalmente tendo como fonte de dados a Nota Fiscal de Consumidor Eletrônica (NFC-e). Diante desse cenário, este artigo objetiva propor uma metodologia para precificação dinâmica de produtos/serviços do dia-a-dia. Além disso, desenvolveu-se uma plataforma inicial, a qual foi validada por uma prova de conceito realizada no estado do Rio Grande do Norte, evidenciando-se sua viabilidade com resultados encorajadores.
\end{abstract}

Keywords: Big Data; Smart Economy; Dynamic Pricing; NFC-e.

Palavras-chaves: Big Data, Economia Inteligente, Precificação Dinâmica, NFC-e.

\section{INTRODUÇÃO}

Cidades Inteligentes, também comumente conhecida por smart cities, são fundações dotadas de recursos tecnológicos para prover qualidade de serviços para seus cidadãos por meio do crescimento econômico e sustentável de sua infraestrutura (Alavi et al., 2018; Anand and Navío-Marco, 2018; Hashem et al., 2016). Dessa forma, smart cities abordam questões tanto críticas quanto do dia-a-dia, tais como gerenciamento de crimes, educação, economia, energia e transportes, com o propósito de fornecer melhores serviços públicos e uso efetivos dos recursos disponíveis. Além disso, uma smart city consiste de seis componentes fundamentais: smart governance, smart economy, smart citizens, smart mobility, smart environment e smart living (Alavi et al., 2018).

Dentre esses elementos essenciais da smart city, destacase a smart economy que, segundo Arroub et al. (2016), apesar de não possuir uma definição universal, é descrita como uma economia inovadora, empreendedora e flexível, bem como é caracterizada pela integração de todos os tipos de mercado. Assim, o smart economy possui como cerne o uso intensivo de tecnologias de comunicação e informação para garantir a competitividade, produtividade, redução de custos e foco no bem-estar dos cidadãos (Arroub et al., 2016).

São vários os recursos tecnológicos disponíveis para promover smart economy, sendo as abordagens de análise e previsão de preços indispensáveis e essenciais para proporcionar uma melhor capacidade analítica do histórico de preços de mercadorias (Ståhl et al., 2018; Tziridis et al., 2017).

Nesse sentido, várias pesquisas têm sido feita nessa linha de estudo — análise e predição de preços — por meio do uso de técnicas de Inteligência Artificial e/ou Machine Learning em diversos contextos: bolsa de valores (Wang et al., 2018), energia elétrica (Lago et al., 2018), ouro, prata e cobre (Liu et al., 2017), petróleo (Tebyanian and Hedayati, 2014), casa/imobiliário (Banerjee and Dutta, 2017) e passagens aéreas (Tziridis et al., 2017).

Contudo, uma lacuna de pesquisa pouco explorada é a utilização dessas técnicas para análise e predição de preços de produtos do dia-a-dia do consumidor, a exemplo de 
combustíveis (gasolina e/ou álcool), itens alimentícios, medicamentos e material de escritório e/ou escolar. Ademais, as fontes de dados geralmente utilizadas nesses estudos são de um domínio específico e, em alguns casos, privadas, além de não contemplarem bens de consumo comumente usados pelas pessoas no seu dia-a-dia. Por outro lado, um recurso pouco abordado nessa linha de pesquisa é a Nota Fiscal de Consumidor Eletrônica (NFC-e), a qual é um documento fiscal cuja finalidade é registrar operações comerciais de vendas realizadas pelos lojistas (ENCAT, 2019).

Diante desse contexto, para promover smart economy em cidades inteligentes, torna-se necessário uma abordagem de precificação dinâmica de produtos e/ou serviços do diaa-dia, a qual possibilitará aos consumidores a busca pelo melhor custo-benefício em suas aquisições e o aumento da competitividade entre empresas, além de permitir a análise de dados oriundos de NFC-es. Assim, este artigo tem como objetivo apresentar uma metodologia proposta para precificação dinâmica de produtos e/ou serviços a partir de NFC-es. Com a finalidade de validar essa abordagem em questão, uma prova de conceito foi realizada no estado do Rio Grande do Norte (RN) e seus resultados foram bastante satisfatórios.

Esse artigo está organizado da seguinte forma: a seção 2 apresenta brevemente a NFC-e. Na seção 3, são discutidos os trabalhos relacionados. A metodologia proposta é explanada na seção 4 . Os resultados são apresentados na seção 5. Finalmente, é discutida, na seção 6 , a conclusão e trabalhos futuros.

\section{NOTA FISCAL DE CONSUMIDOR ELETRÔNICA (NFC-E)}

Como dito anteriormente, NFC-e é o documento fiscal emitido e armazenado eletronicamente, com o propósito de documentar operações comerciais de vendas ao consumidor final em operação interna e sem geração de crédito de ICMS ao adquirente (Secretaria da Tributação do Estado de Rio Grande do Norte, 2019; ENCAT, 2019).

Assim, a NFC-e possui várias informações passíveis de análise (ver Figura 1), tais como detalhes do emitente (vendedor/lojista), detalhamento dos produtos/serviços (descrição, código, quantidade, preço), totais que serão impressos no Documento Auxiliar da Nota Fiscal Eletrônica (DANFE) NFC-e (resumo da compra e formas de pagamentos), dados referentes à consulta via chave de acesso e por $Q R$ Code, dados do comprador (opcional), entre outros (ENCAT, 2018).

Além disso, conforme a Secretaria da Tributação do Estado de Rio Grande do Norte (2019), dentre as diversas vantagens da NFC-e, destacam-se: transmissão em tempo real ou online de suas informações; uso de novas tecnologias de mobilidade; integração de plataformas de vendas físicas e virtuais; possibilidade de consulta (resumida ou completa) em tempo real ou online de suas NFC-e no portal da SEFAZ; e segurança quanto à validade e autenticidade da transação comercial.

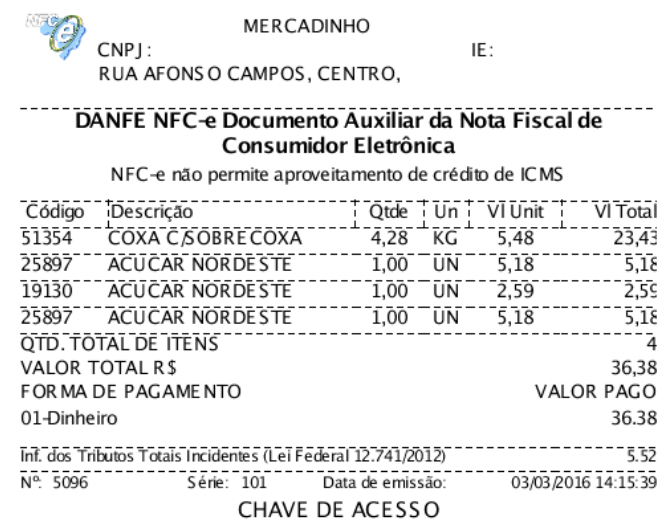

00-0000-00.000.000/0000-00-00-000-000.000.000-000.000.000-0

\section{CONSUMIDOR}

CNPJ /CPF AD Estrangeiro
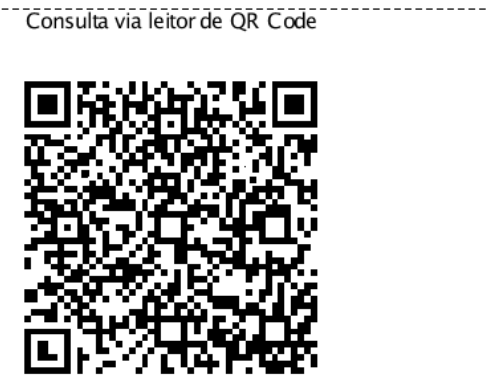

Protocolo de Autorização: 03/03/2016 14:15:46

Figura 1. Exemplo de NFC-e.

\section{TRABALHOS RELACIONADOS}

Diante dos diversos estudos encontrados, que atuam na área de análise e predição de preços de produtos e/ou serviços, destacam-se os trabalhos abaixo.

Tebyanian and Hedayati (2014) propuseram dois ensembles de algoritmos de regressão para previsão do preço diário de petróleo a partir de dados da Administração de Energia dos EUA e de algumas agências internacionais de notícias. Ambos os modelos ensembles foram avaliados com os algoritmos de Regressão Linear, Árvores de Decisão, Processos Gaussianos e Regressão de Vetor de Suporte (SVR). Ao final dos experimentos, o ensemble "pesados exponencialmente" com SVR obteve o melhor desempenho.

Para analisar a predição de preços de passagens aéreas, Tziridis et al. (2017) avaliaram o desempenho de oito técnicas de Machine Learning consagradas pela literatura: Multilayer Perceptron (MLP), Generalized Regression Neural Network, Extreme Learning Machine (ELM), Random Forest Regression Tree, Árvore de Regressão, Bagging de Árvore de Regressão, Regressão de Vetor de Suporte (Polinomial e Linear) e Regressão Linear. Ao final dos experimentos, constatou-se que os modelos preditivos eram adequados para tratar esse problema de regressão, apresentando acurácia acima de $88 \%$.

Banerjee and Dutta (2017) utilizaram técnicas de Machine Learning para realizar predições a respeito do aumento ou queda nos preços de casas/imóveis. Os algoritmos usados foram: Rede Neural Artificial (ANN), Máquina de Vetor de Suporte (SVM) e Random Forest. Após a finalização 
dos experimentos, o desempenho do SVM foi superior em relação aos outros algoritmos.

Por fim, Aujla et al. (2018) definiram um esquema de precificação dinâmica de energia de pontos de carga para veículos elétricos, o qual era uma abordagem multiparametrizada que considerava o percentual de carga, tempo de uso, localização e tipo de veículo elétrico. Tal esquema se baseava nos dois casos do Jogo de Stackelberg - (a) os veículos elétricos como líderes e os pontos de cargas como seguidores, e (b) os pontos de carga como líderes e os veículos como seguidores. Os autores avaliaram o modelo proposto com três tipos de veículos com relação às métricas de desempenho - preço da energia, função de utilidade e fator de satisfação. Após a realização dos experimentos, a abordagem multi-parametrizada obteve desempenho superior em relação aos esquemas existentes (por preço fixo e por tempo de uso).

Os trabalhos correlatos apresentados retratam o uso de técnicas de Inteligência Artificial e/ou Machine Learning para análise e previsão de preços em contextos específicos. Assim, o diferencial deste estudo é uma metodologia de precificação dinâmica de produtos/serviços do dia-a-dia a partir dos dados contidos em NFC-es, contemplando desde a coleta, tratamento e armazenamento das informações das notas fiscais até a análise e previsão dinâmica de preços.

\section{METODOLOGIA}

Para Anand and Navío-Marco (2018), alguns dos principais desafios de smart cities são: (I) a maneira como gerar, coletar e analisar dados significantes; (II) como garantir a segurança dos dados e privacidade dos cidadãos; e (III) como desenvolver uma arquitetura que permita a comunicação entre diversos sistemas e stakeholders.

Outrossim, Anand and Navío-Marco (2018) complementa que ainda são necessários projetos, abordagens e metodologias para smart cities que permitam a colaboração efetiva entre cidadãos e que contemplem os quatros aspectos/dimensões da smart economy, a saber: (I) a escala de mercado global; (II) os dados a serem utilizados; (III) a estrutura e competitividade do mercado; e (IV) o impacto na economia local.

Partindo desse pressuposto, foi proposta uma metodologia de precificação dinâmica para produtos e serviços comuns ao dia-a-dia de cidadãos, no contexto de smart cities, a partir dos dados de NFC-es. Uma visão geral da abordagem proposta é apresentada na Figura 2.

Como pode ser visto na Figura 2, toda compra de um produto ou contratação de serviço efetuada por um cliente gera uma NFC-e, possuindo informações de alta potencialidade de análise, tais como descrição e preços de produtos/serviços, dados do lojista, formas de pagamento utilizadas, entre outras. Dessa forma, pode-se considerar os cidadãos, juntamente com seus smartphones, como sensores (people as sensors), possibilitando a coleta e transmissão desses dados.

Assim, uma plataforma inicial foi desenvolvida. A partir de sua aplicação Android, é possível realizar a leitura de uma NFC-e por meio de seu $Q R$ Code e, em seguida, é encaminhado o URL (Uniform Resource Locator) da página-espelho dessa nota para o servidor Web. Este, por sua vez, é responsável por extrair dados relevantes da nota fiscal com uso de Web Scraping, além de persistir tais informações em um repositório, o qual possibilitará análises e predições posteriores. Também a abordagem em questão fornecerá feedback personalizado ao usuário, baseado em suas interações com a plataforma, agregando assim valor ao cidadão a partir do conhecimento/informação retornada, a exemplo de um resumo categorizado e previsão de seus gastos.
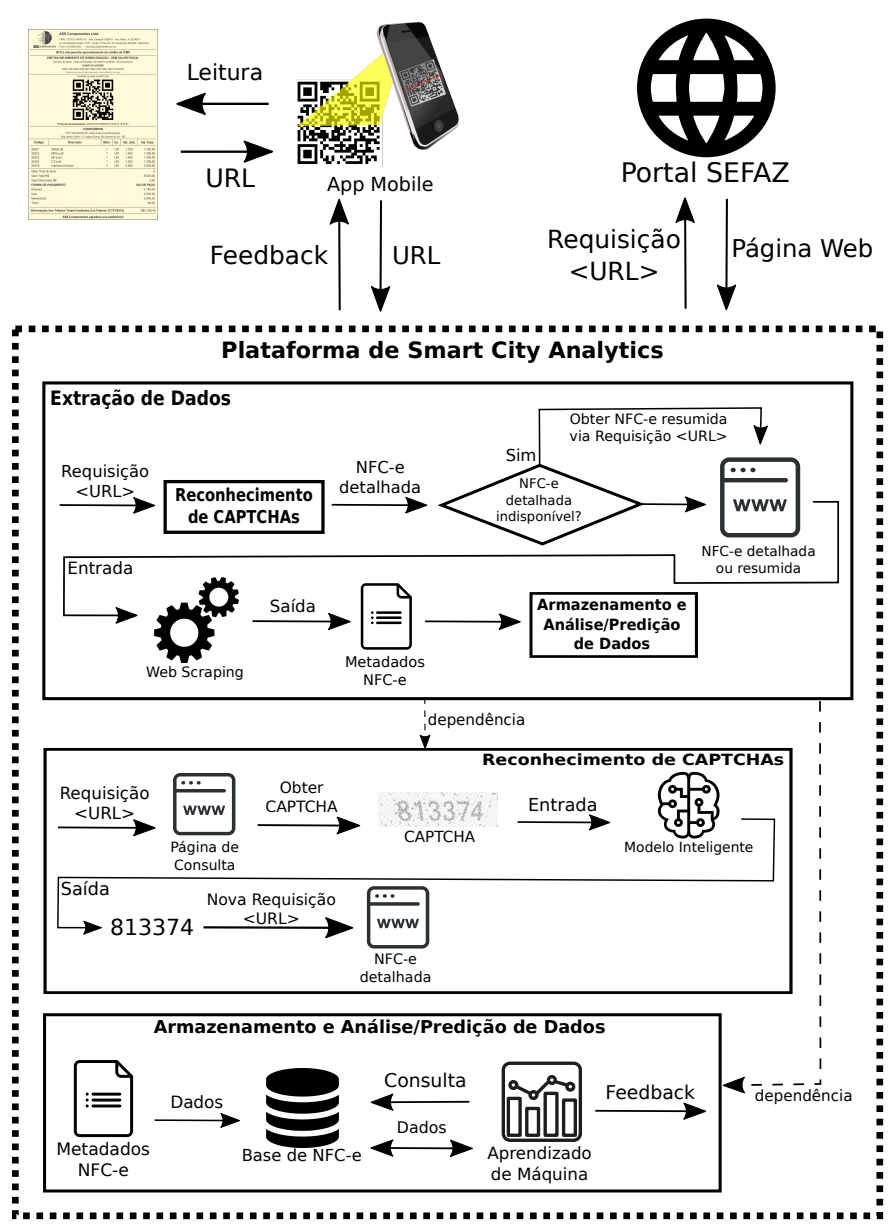

Figura 2. Metodologia para precificação dinâmica de produtos e serviços em Cidades Inteligentes.

Por intermédio dos dados contidos nesse repositório e da plataforma, cidadãos e empresas usufruirão de muitos benefícios, sendo alguns deles:

- Busca do melhor custo-benefício na aquisição de produtos, baseado em sua localização atual;

- Estímulo de consumo para obtenção de descontos em organizações (uso de gamificação);

- Previsão de preços de produtos e serviços com base em dados históricos;

- Detecção de padrões a partir dos comportamentos de consumo dos cidadãos.

Após um overview da metodologia proposta, será explanado, a seguir, em detalhes, o processo de Web Scraping para extração de dados contidos em NFC-es. 


\subsection{Processo de Web Scraping para NFC-es}

Como visto anteriormente, existem duas formas de consulta à NFC-e: simplificada/resumida e completa. Também é possível o acesso a partir do $Q R$ Code da nota fiscal, contudo, perde-se algumas características e informações detalhadas dos produtos/serviços, a exemplo dos códigos de barras e GTIN (Global Trade Item Number), uma vez que esse meio de visualização é do tipo sumarizado.

Desse modo, uma forma de contornar esse problema consiste em realizar Web Scraping na página de consulta de NFC-es. Com esse propósito, na abordagem supracitada, utilizou-se Web Scraping juntamente com um mecanismo de reconhecimento automático de CAPTCHAs, o qual é baseado em técnicas de processamento de imagens e aprendizado de máquina, possibilitando-o inferir o código pertencente à imagem.

Para fins experimentais, essa metodologia foi validada por meio de uma prova de conceito realizada na página de consulta da Secretaria de Tributação do Estado do Rio Grande do Norte $(\mathrm{SET} / \mathrm{RN})^{1}$, tendo como campos obrigatórios a chave de acesso contida na NFC-e e seu CAPTCHA, como mostrado na Figura 3.

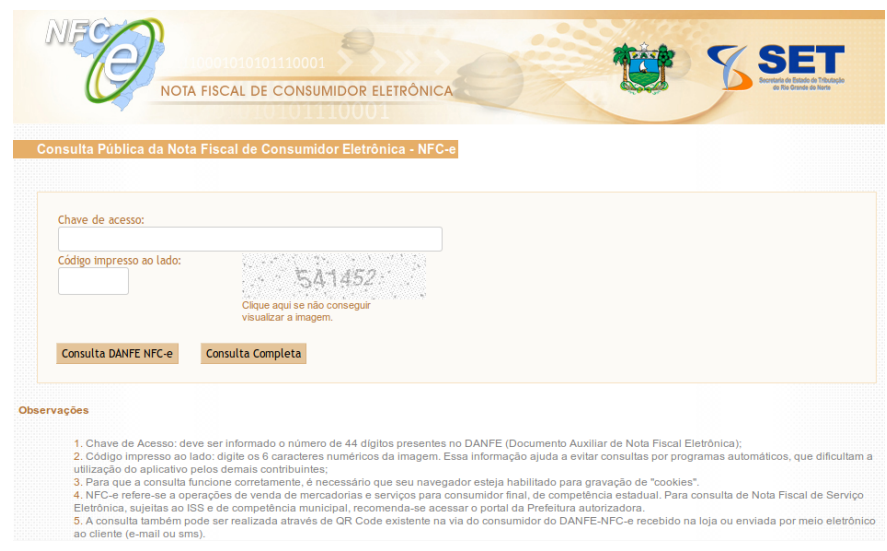

Figura 3. Página de Consulta de NFC-e da SET/RN.

Assim, 1000 imagens de CAPTCHAs foram coletadas da página de consulta do $\mathrm{SET} / \mathrm{RN}$ para compor os conjuntos de treinamento e de teste do sistema de reconhecimento. Nessa coleta, percebeu-se que os CAPTCHAs utilizados pelo SET/RN limitam-se apenas a seis números, sendo que cada dígito pode assumir um valor entre zero e oito.

A seguir, serão apresentados os módulos do mecanismo de identificação, a saber: segmentação e reconhecimento ótico de caracteres (OCR).

Segmentação de Caracteres. Para realizar a segmentação dos caracteres, utilizou-se a linguagem Python ${ }^{2}$ e OpenCV ${ }^{3}$, biblioteca open source de visão computacional. Inicialmente, converte-se a imagem de espaço de cores RGB em escala de cinza (OpenCV, 2019), de acordo com seguinte equação:

$$
f_{g}(I, i, j)=I_{i j}^{R} \cdot 0.299+I_{i j}^{G} \cdot 0.587+I_{i j}^{B} \cdot 0.114
$$

\footnotetext{
1 https://nfce.set.rn.gov.br/portalDFE/NFCe/

ConsultaNFCe.aspx

2 https://www.python.org/

3 https://opencv.org/
}

onde: $I$ é a imagem a ser convertida; $i j$ as coordenadas de linha e coluna; $I_{i j}^{R}, I_{i j}^{G}$ e $I_{i j}^{B}$ os valores dos pixels nas posições ij dos canais $R, G$ e $B$, respectivamente (ver Figura 4a).

Em sequência, é aplicado uma limiarização, com o intento de transformar a imagem em escala de cinza em binária, conforme a seguinte fórmula:

$$
T(I, i, j, k)= \begin{cases}255 & \text { se } I_{i j} \geq k \\ 0 & \text { caso contrário }\end{cases}
$$

onde: $I$ é a imagem a ser convertida; $i j$ as coordenadas de linha e coluna; e $k$ o limiar de transformação. A Figura 4b exemplifica o resultado obtido pela aplicação de $T$.

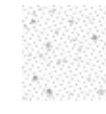

(a)

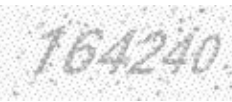

CAPTCHA em escala de cinza

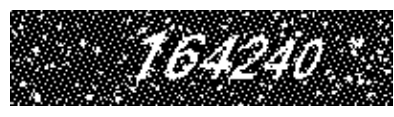

(b)
CAPTCHA após a limiarização
Figura 4. Exemplos de CAPTCHAs em escala de cinza e limiarizada.

Após essa transformação, aplica-se uma sequência de filtros de mediana, em concordância com as seguintes fórmulas:

$$
\begin{gathered}
\text { Slice }\left(I, C_{x}, C_{y}, S\right)=\left[\begin{array}{ccccc}
I_{C_{y}-S / 2}^{C_{x}-S / 2} & \cdots & I_{C_{y}}^{C_{x}-S / 2} & \cdots & I_{C_{y}+S / 2}^{C_{x}-S / 2} \\
\vdots & \ddots & \vdots & \ddots & \vdots \\
I_{C_{y}-S / 2}^{C_{x}} & \cdots & I_{C_{y}}^{C_{x}} & \cdots & I_{C_{y}+S / 2}^{C_{x}} \\
\vdots & \ddots & \vdots & \ddots & \vdots \\
I_{C_{y}+S / 2}^{C_{x}+S / 2} & \cdots & I_{C_{y}}^{C_{x}+S / 2} & \cdots & I_{C_{y}+S / 2}^{C_{x}+S}
\end{array}\right] \\
\operatorname{Med}(I, S)=\operatorname{median}\left(I_{i j}\right), K=\{0, \cdots, S / 2\} \\
\quad i, j \in K \\
M\left(I, C_{x}, C_{y}, S\right)=\operatorname{Med}\left(\operatorname{Slice}\left(I, C_{x}, C_{y}, S\right), S\right)
\end{gathered}
$$

onde: $S$ é o tamanho do recorte a ser realizado na imagem; Slice a função responsável por retornar um recorte da imagem $I$ com tamanho $S \times S$ e centros $C_{x}$ e $C_{y} ; M e d$ retorna o valor da mediana de uma matriz $I$ de tamanho $S \times S$; e $M$ é a função que, para cada coordenada de linha e coluna da imagem $I$, a mediana dessa posição é calculada por meio de $M e d$, considerando um recorte de tamanho $S \times S$. A Figura 5 mostra a aplicação da sequência de filtros de mediana, utilizando um recorte $3 \times 3$.

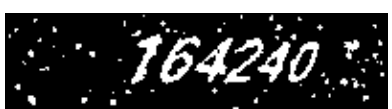

(a)

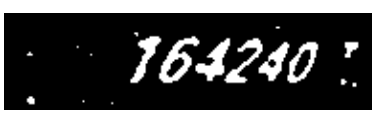

(c) $\quad 3^{\mathrm{a}}$ aplicação

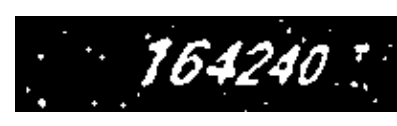

(b) $2^{\mathrm{a}}$ aplicação

\section{0}

(d) $4^{\mathrm{a}}$ aplicação
Figura 5. Sequência de filtros de mediana.

Por fim, os dígitos são encontrados por intermédio de um algoritmo de detecção de contornos (Suzuki and Abe, 
1985), o qual utiliza imagens binárias para detectar bordas a partir de fronteiras, em que os seis mais representativos — baseados na quantidade de pixels - são inferidos como possíveis dígitos do CAPTCHA (ver Figura 6).

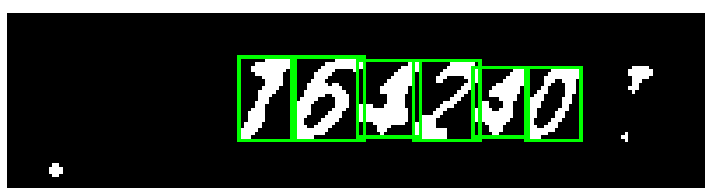

Figura 6. CAPTCHA com dígitos segmentados.

Reconhecimento Ótico de Caracteres (OCR). Para realizar o OCR dos dígitos de um CAPTCHA, utilizouse o algoritmo de aprendizado supervisionado Multi-layer Perceptron (MLP) como método de reconhecimento. Para essa etapa, é necessário transformar cada dígito da imagem em um vetor característica, sendo redimensionado para $15 p x \times 20 p x$ e, posteriormente, transformado em um vetor coluna $300 \times 1$.

Por fim, esse vetor de característica é utilizado como entrada da rede neural, a qual tem como resposta um dígito entre 0 a 8 . Portanto, para cada dígito, esse processo é repetido, resultando em uma lista com os caracteres do CAPTCHA inferidos pelo modelo.

\section{EXPERIMENTOS E RESULTADOS}

Inicialmente, foi aplicado a segmentação nos dígitos dos 1000 CAPTCHAs supracitados; em sequência, esses números foram categorizados em 9 classes (ver Tabela 1). Dessa forma, obteve-se um total de 5262 de dígitos válidos — por não estarem deformados ou cortados - , enquanto que os demais foram considerados como ruídos ou inapropriados. Ainda nessa fase, dividiu-se os dígitos segmentados em dois conjuntos, treinamento e testes, com $80 \%$ e $20 \%$ dos dados, respectivamente. Também criou-se um classificador cuja configuração é apresentada na Tabela 2.

Tabela 1. Dígitos segmentados por classe.

\begin{tabular}{cc}
\hline Classe & Quantidade \\
\hline 0 & 602 \\
1 & 531 \\
2 & 574 \\
3 & 613 \\
4 & 563 \\
5 & 585 \\
6 & 566 \\
7 & 640 \\
8 & 588 \\
ruído & 738 \\
\hline Total & $\mathbf{6 0 0 0}$ \\
\hline
\end{tabular}

Tabela 2. Hiperparâmetros da MLP.

\begin{tabular}{lc}
\hline Hiperparâmetros & Valor \\
\hline Camadas escondidas & 2 \\
Neurônios & 100 \\
Função de ativação & relu \\
Solucionador & adam \\
Taxa de aprendizado & 0.0001 \\
Iterações máximas & 1000 \\
Momento & 0.9 \\
\hline
\end{tabular}

A Tabela 3 apresenta os resultados da rede neural a partir do conjunto de testes, os quais são bastante satisfatórios, além de evidenciarem a eficácia do modelo.

Tabela 3. Resultados da avaliação da MLP.

\begin{tabular}{lc}
\hline Métrica & $\%$ \\
\hline Acurácia & 99.05 \\
Precisão & 99.05 \\
Recall & 99.06 \\
F1-score & 99.04 \\
\hline
\end{tabular}

Ademais, como dito anteriormente, uma prova de conceito foi realizada com o intuito de analisar a viabilidade da abordagem proposta, sendo utilizada para acessar a plataforma do SET/RN de forma automática. Dessa maneira, foram feitas 1100 tentativas de acesso ao referido sistema, obtendo sucesso em 673 delas, ou seja, a solução em questão conseguiu obter os dados de uma NFC-e em 61,18\% das chances. Em outras palavras, a plataforma proposta precisa, em média, de 1.63 investidas para acessar com sucesso uma nota fiscal, com desvio padrão igual a \pm 1.02, comprovando a factibilidade de implementação dessa solução, uma vez que nem sempre será necessário realizar mais de uma tentativa.

Complementando a prova de conceito, a partir de uma amostra de notas fiscais de compras realizadas em quatro varejistas do $\mathrm{RN}$, realizou-se uma análise de dados sobre os dados contidos nessa amostra. Assim, identificou-se as 10 principais categorias de produtos e seus preços médios, com destaque para itens alimentícios (arroz, leite em pó, macarrão, entre outros), como é mostrado na Figura 7.

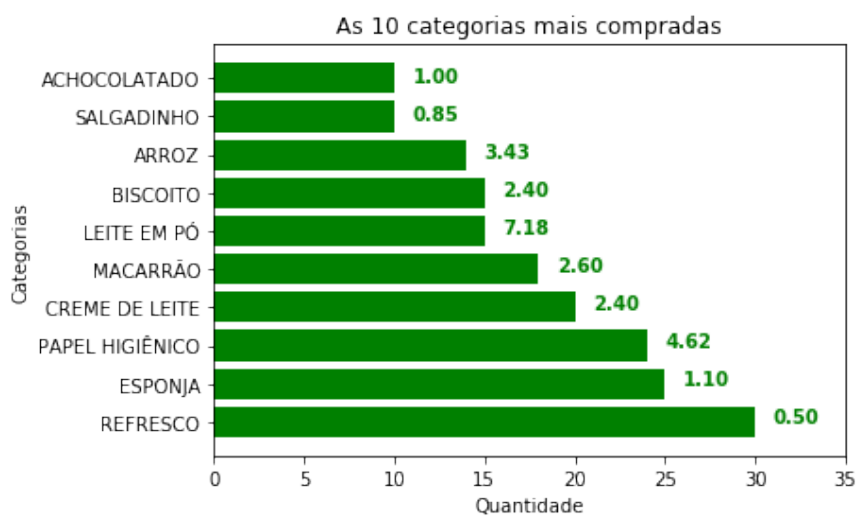

Figura 7. Categorias de produtos mais vendidas e seus preços médios.

\section{CONCLUSÃO}

Smart economy é consensualmente definida como uma economia inovadora e integrada com o propósito de proporcionar maior competitividade, produtividade e custobenefício para os cidadãos de smart cities. Para tal fim, diversas abordagens têm sido desenvolvidas, destacandose aquelas relacionadas à análise e previsão de preços.

Contudo, esses mecanismos são poucos explorados no contexto de produtos/serviços do dia-a-dia de cidadãos, principalmente tendo como fonte de dados as NFC-es. Diante desse cenário, neste estudo, foi proposta uma metodologia para precificação dinâmica para a conjuntura supracitada. 
Ademais, desenvolveu-se uma plataforma inicial, a qual foi validada por uma prova de conceito.

Embora seja um estudo inicial, os resultados são encorajadores, evidenciando a viabilidade da metodologia e plataforma inicialmente desenvolvida.

Como trabalhos futuros, é de suma importância o refinamento da arquitetura da plataforma, utilização de técnicas de processamento de linguagem natural para padronização/unificação das descrições dos produtos/serviços, definição do modelo inteligente para análise e previsão de preços - com base no estado da arte -, escolha do mecanismo de privacidade e segurança dos dados Blockchain para segurança de dados, por exemplo - e, por fim, a realização de novos experimentos.

\section{REFERÊNCIAS}

Alavi, A.H., Jiao, P., Buttlar, W.G., and Lajnef, N. (2018). Internet of things-enabled smart cities: State-of-the-art and future trends. Measurement, 129, 589-606.

Anand, P.B. and Navío-Marco, J. (2018). Governance and economics of smart cities: opportunities and challenges.

Arroub, A., Zahi, B., Sabir, E., and Sadik, M. (2016). A literature review on smart cities: Paradigms, opportunities and open problems. In 2016 International Conference on Wireless Networks and Mobile Communications (WINCOM), 180-186. IEEE.

Aujla, G.S., Kumar, N., Singh, M., and Zomaya, A.Y. (2018). Energy trading with dynamic pricing for electric vehicles in a smart city environment. Journal of Parallel and Distributed Computing.

Banerjee, D. and Dutta, S. (2017). Predicting the housing price direction using machine learning techniques. In 2017 IEEE International Conference on Power, Control, Signals and Instrumentation Engineering (ICPCSI), 2998-3000.

ENCAT (2018). Manual de Especificações Técnicas do $D A N F E N F C$-e e QR Code - Versão 5.0. ENCAT. URL https://bit.1y/2Ct7vcH. Acessado em: 06 jan. 2019.

ENCAT (2019). O que é nfc-e? URL https://bit.ly/ 2uk72Fr. Acessado em: 06 jan. 2019.

Hashem, I.A.T., Chang, V., Anuar, N.B., Adewole, K., Yaqoob, I., Gani, A., Ahmed, E., and Chiroma, H. (2016). The role of big data in smart city. International Journal of Information Management, 36(5), 748-758.

Lago, J., Ridder, F.D., and Schutter, B.D. (2018). Forecasting spot electricity prices: Deep learning approaches and empirical comparison of traditional algorithms. Applied Energy, 221, 386-405. doi:https://doi.org/10. 1016/j.apenergy.2018.02.069.

Liu, C., Hu, Z., Li, Y., and Liu, S. (2017). Forecasting copper prices by decision tree learning. Resources Policy, 52, 427-434. doi:https://doi.org/10.1016/j.resourpol. 2017.05.007.

OpenCV (2019). Color conversions. URL https://bit. ly/2PbY5Yi. Acessado em: 06 jan. 2019.

Secretaria da Tributação do Estado de Rio Grande do Norte (2019). A nfc-e. URL https://bit.1y/2TnbBsv. Acessado em: 06 jan. 2019.

Ståhl, J.E., Windmark, C., and Kianian, B. (2018). Costbased pricing for learning organizations - a model presentation and demonstration. Procedia Manufacturing, 25, 239-246. doi:https://doi.org/10.1016/j.promfg.2018.
06.079. Proceedings of the 8th Swedish Production Symposium (SPS 2018).

Suzuki, S. and Abe, K. (1985). Topological structural analysis of digitized binary images by border following. Computer Vision, Graphics, and Image Processing, 30(1), 32-46. doi:https://doi.org/10.1016/ 0734-189X (85)90016-7.

Tebyanian, A. and Hedayati, F. (2014). Intelligent crude oil price forecaster. In 2014 13th International Conference on Machine Learning and Applications, 453-455. doi:10.1109/ICMLA.2014.79.

Tziridis, K., Kalampokas, T., Papakostas, G.A., and Diamantaras, K.I. (2017). Airfare prices prediction using machine learning techniques. In 2017 25th European Signal Processing Conference (EUSIPCO), 1036-1039.

Wang, Q., Xu, W., and Zheng, H. (2018). Combining the wisdom of crowds and technical analysis for financial market prediction using deep random subspace ensembles. Neurocomputing, 299, 51-61. doi:https://doi.org/ 10.1016/j.neucom.2018.02.095. 